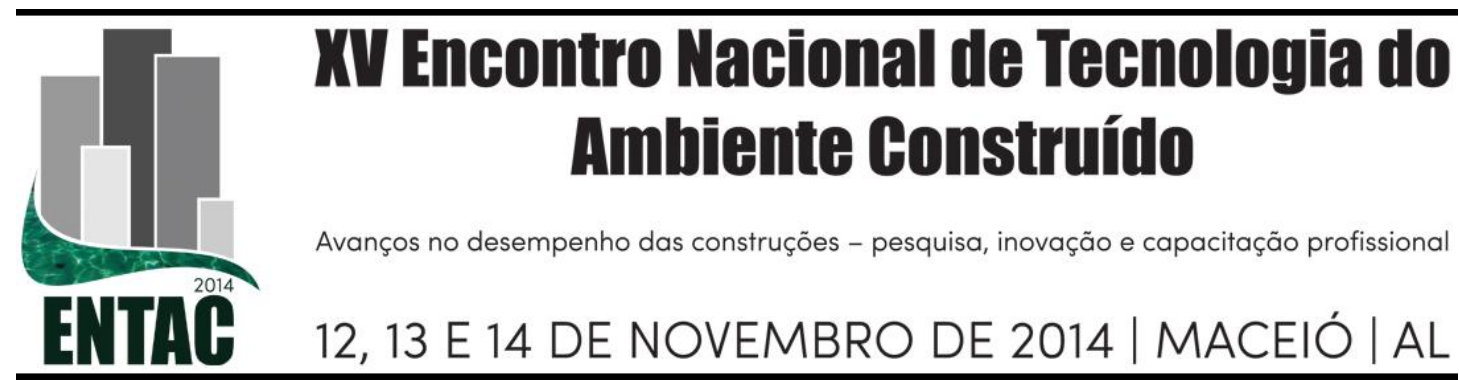

\title{
INFLUÊNCIA DO NÚMERO DE DIVISÓRIAS DOS CAPTADORES DE VENTO NA VENTILAÇÃO NATURAL DE EDIFICAÇÕES
}

\author{
ANDRADE, Nixon Cesar (1); LABAKI, Lucila Chebel (2); CARAM, Rosana \\ Maria (3); GRECO-JUNIOR, Paulo Celso (4)
}

(1) UNICAMP, (16) 33723204, deandraden@bol.com.br, (2) UNICAMP, (19) 35212384, llabaki@gmail.com, (3) USP, (16) 33739311, rcaram@sc.usp.br, (4) USP, (16) 33738124, pgreco@sc.usp.br

\begin{abstract}
RESUMO
Uma das alternativas existentes para promover a ventilação natural dos edifícios é a utilização de torres de vento situadas acima do nível da cobertura, as quais podem funcionar como captadores ou extratores de ar. Esse artigo tem como objetivo analisar dois tipos de torres de vento, atuando como captadores: a octodirecional e a dodecadirecional, com oito e doze aberturas respectivamente, variando o número de suas divisórias, por meio de simulações no software $\mathrm{CFX}^{\circledR}$, as quais permitiram estudar as características técnicas dessas torres e seus efeitos sobre a ventilação interna do ambiente. Trata-se de uma pesquisa desenvolvida no doutorado, acrescida com outras simulações para verificação. Classifica-se, quanto aos objetivos, como um estudo exploratório. A metodologia foi dividida nas seguintes etapas: construção da geometria, na qual as torres octo e dodecadirecional foram projetadas com oito e doze divisórias, respectivamente, e, ambas com quatro divisórias; definição das parts; determinação da malha; condições de contorno e controle de solução no CFX Pre ${ }^{\circledR}$; processamento das simulações no CFX Solver ${ }^{\circledR}$; visualizações dos resultados no CFX Post ${ }^{\circledR}$ : velocidade e coeficiente de pressão. Os resultados obtidos por uma série de simulações computacionais permitiram a análise e comparação dos projetos propostos, auxiliando na determinação das configurações mais adequadas, concluindo que as torres octodirecional e dodecadirecional com quatro divisórias são as mais eficientes, pois proporcionam maiores valores de velocidade do ar interno na maioria das posições consideradas. Desta forma, utilizando o software $\mathrm{CFX}^{\circledR}$, as análises possibilitaram desenvolver melhorias para os ambientes avaliados, no que se refere ao desempenho da ventilação, que proporcionará melhores taxas de conforto aos usuários da edificação, permitindo verificar as aberturas mais adequadas para captação e distribuição do vento no interior dos ambientes.
\end{abstract}

Palavras-chave: Captador de vento, Torre de vento, Ventilação natural, Arquitetura bioclimática, $\mathrm{CFX}^{\circledR}$.

\begin{abstract}
The use of wind towers located above the roof is an alternative for the promotion of natural ventilation in buildings. Such towers can operate as either catchers or air extractors. This paper, ranked as an exploratory study regarding its objectives, analyzes two types of wind towers that act as catchers: one octodirectional and another dodecadirectional, with eight and twelve openings, respectively. The number of their partitions was varied by simulations in $C F X{ }^{\circledR}$ software, which enabled the study of the technical characteristics of the towers and their effects on indoor ventilation. The methodology consists of the following steps: construction of the geometry, where the octa and dodecadirecional towers were designed with eight and twelve partitions, respectively, and two with four partitions; definition of parts; determination of the mesh; boundary conditions and solution control in CFX Pre ${ }^{\circledR}$; simulations in CFX Solver $^{\circledR}$; visualization of the results in CFX Post ${ }^{\circledR}$ : determination of the speed and pressure coefficient. The results of a series of computer simulations enabled the analysis and comparison of the proposed projects and assisted in the determination of the most appropriate settings. The octodirectional and dodecadirectional towers with four partitions showed the most efficient, as they provided a higher rate of indoor air in most positions considered. The analysis with CFX ${ }^{\circledR}$ software enabled improvements in the
\end{abstract}


environments evaluated regarding the performance of ventilation, which will provide favorable exchange comfort rates to the users of the building for the verification of the most appropriate openings for the capture and distribution of wind within the environment.

Keywords: Wind catcher, Wind tower, Natural ventilation, Bioclimatic architecture, CFX ${ }^{\circledR}$.

\section{INTRODUÇÃOO}

A ventilação natural é uma das estratégias mais adequadas para o resfriamento passivo dos edifícios, por isso a massa de ar no interior dos ambientes deve ser continuamente retirada e substituída por ar fresco de uma fonte externa e limpa para resfriar a edificação e/ou direcionar sobre os usuários com o objetivo de tornar os ambientes termicamente confortáveis.

Uma das alternativas existentes para se alcançar tal proposição é a utilização de torres de vento situadas acima do nível da cobertura, as quais podem funcionar como captadores ou extratores de ar. Estudos qualitativos recentes, sobre o padrão de circulação do vento no interior da tipologia examinada, demonstraram que tais dispositivos podem ser eficientes como solução arquitetônica na promoção da renovação do ar e do conforto higrotérmico.

Esse artigo tem como objetivo analisar dois tipos de torres de vento, atuando como captadores: a octodirecional e a dodecadirecional, com oito e doze aberturas respectivamente, variando o número de suas divisórias, por meio de simulações no software $\mathrm{CFX}^{\circledR}$.

As torres de vento, desde que posicionadas corretamente para a captação dos ventos dominantes, podem contribuir para um aumento significativo da ventilação natural das edificações, cujos benefícios proporcionados são o conforto térmico e a economia de energia elétrica.

\section{REVISÃO BIBLIOGRÁFICA}

Martins et al (2009) afirmam que em grande parte da região do Brasil, onde predomina o clima quente e úmido, as taxas de velocidade do ar são bastante altas e passíveis de serem amplamente utilizadas para proporcionar conforto por meio do uso de elementos de captação do vento em edificações. Apesar de ainda pouco empregadas, as torres de vento oferecem um eficiente aproveitamento desse recurso.

\subsection{Definição}

Segundo Bahadori (1994), as torres funcionando como captadores de vento ou "BaudGeers" (em persa) consistem em torres projetadas para "capturar" o vento, que passa acima da cobertura das edificações, e redirecioná-lo para os espaços interiores de uma edificação (Figura 1).

\subsection{Tipologia}

Segundo Van Lengen (2004), as partes que compõem uma torre de vento são (Figura 2):

Base: é formada pelas paredes externas inferiores, sendo que para torres altas elas são mais grossas para apoiar o peso das paredes superiores.

Paredes Cruzadas: a base das paredes cruzadas apoia-se nas paredes externas e são de vigas de madeira ou de concreto. As paredes cruzadas são levantadas sobre essa base.

Aberturas: são formadas a partir do levantamento das paredes externas superiores. 
Teto: ao final a torre recebe uma cobertura horizontal, que pode ser de laje de concreto ou uma abóbada.

Figura 1 - Exemplos de construções com torre de vento

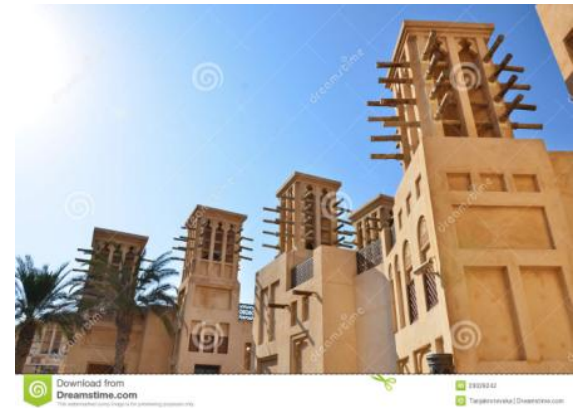

Fonte: http://interata.squarespace.com/jornal-deviagens/2007/8/25/dubai-sheikh-saeed-almaktoum-house.html

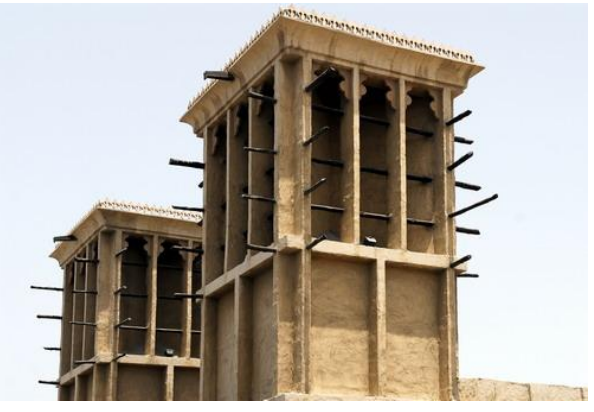

Fonte: http://pt.dreamstime.com/fotografia-destock-torres-do-vento-em-dubai-uaeimage23029242

Figura 2 - Componentes de uma torre de vento

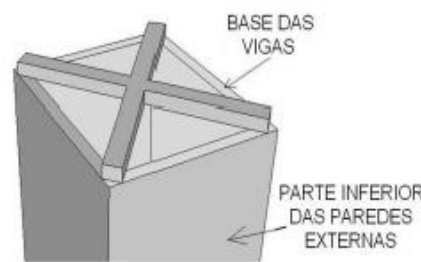

Base com vigas cruzadas

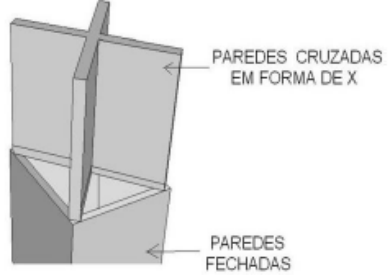

Base com paredes cruzadas

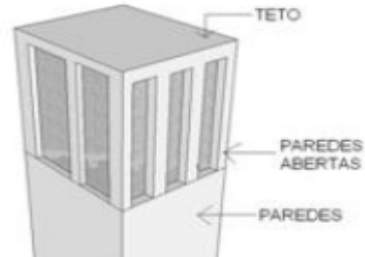

Torre com as aberturas e teto

Fonte: Adaptado de Van Lengen (2004)

Em consideração ao número de aberturas, Bahadori, Mazidi e Dehghani (2008), afirmam que o ar ao entrar em uma torre com mais de uma abertura tem uma parte perdida pelas outras aberturas, enquanto que na torre unidirecional, apesar de pouco utilizada, todo o ar é aproveitado para ventilar a edificação.

\subsection{Pressão de Velocidade}

Segundo Liddament (1986), quando uma corrente livre atinge um obstáculo, a pressão real do vento, num dado ponto do obstáculo, é o produto da pressão dinâmica por um coeficiente de correlação chamado de coeficiente de pressão $(\mathrm{Cp})$.

Os coeficientes de pressão dependem da forma, da porosidade do edifício e da direção do vento. Eles são determinados experimentalmente, em geral, por meio de testes em túnel de vento e são adimensionais:

$$
C p=\frac{p-p_{0}}{\frac{1}{2} \rho V_{0}^{2}} \quad \text { (a) ou } \quad C p=\frac{p-p_{\text {ref }}}{p_{\text {dinnâmiea }}}
$$

onde é o coeficiente de pressão no ponto considerado (adimensional), a pressão no ponto considerado (em $\mathrm{Pa}$ ), a pressão estática em um ponto do escoamento não perturbado pelo modelo físico (em $\mathrm{Pa}$ ), a densidade do ar no interior do túnel de vento 
$\left(\mathrm{em} \mathrm{kg} / \mathrm{m}^{3}\right), V_{0}$ a velocidade do ar em um ponto de escoamento não perturbado pelo modelo físico $(\mathrm{em} \mathrm{m} / \mathrm{s})$, a pressão de referência (em Pa) e a pressão dinâmica (em $\mathrm{Pa})$.

\subsection{CFD (Computational Fluid Dynamics)}

Para Fortuna (2000) o método de simulação numérica aplicado às áreas de Mecânica dos Fluidos e Transferência de Calor, conhecido por CFD ("Computacional Fluid Dynamic") ou DFC ("Dinâmica de Fluídos Computacional") é a área da computação científica que estuda métodos computacionais para simulação de fenômenos que envolvem fluidos em movimento com ou sem trocas de calor.

Segundo Oliveira, Mariano e Quadri (2009) o pacote computacional ANSYS CFX ${ }^{\circledR}$ é um software de simulação para resolução numérica de problemas envolvendo mecânica dos fluidos e transferência de calor (CFD). O programa emprega a metodologia de volumes finitos utilizando estruturas espaciais e gerando malhas correspondentes.

\section{MÉTODO}

Tratando-se de uma pesquisa desenvolvida no doutorado, acrescida com outras simulações para verificação, classifica-se, também, quanto aos objetivos, como um estudo exploratório, cuja metodologia será descrita a seguir.

\subsection{Software ICEM ${ }^{\circledR}$}

\section{Descrição da geometria}

$\mathrm{O}$ modelo da geometria e do domínio computacional para $\mathrm{CFX}^{\circledR}$ foi confeccionado no AutoCAD $^{\circledR}$. No caso, foram construídas duas edificações com torres de geometria octogonal, sendo uma torre com oito e outra com quatro divisórias internas, e, duas edificações com torres de geometria dodecagonal, sendo uma torre com doze e outra com quatro divisórias internas, todas com a cobertura em lajes, e as aberturas estão presentes nas torres e na parede frontal da edificação. Os detalhes da geometria do edifício devem ser considerados, senão muitas interferências deixam de ser respeitadas, alterando os resultados (Figura 3).

Figura 3 - Esquema das edificações com torres octo e dodecadirecional

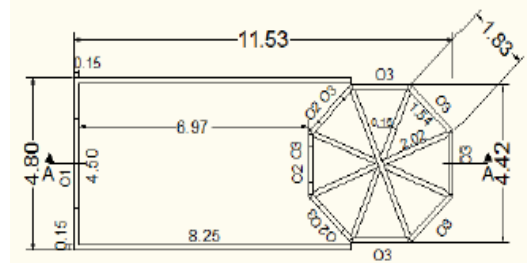

PLANTA COM OITO DIVISÓRIAS

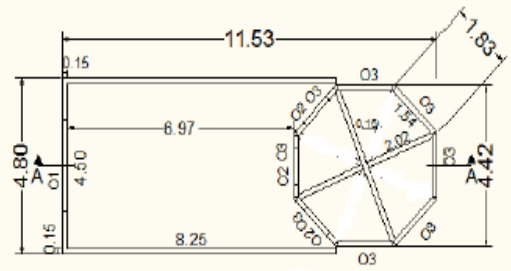

PLANTA COM QUATRO DIVISÓRIAS

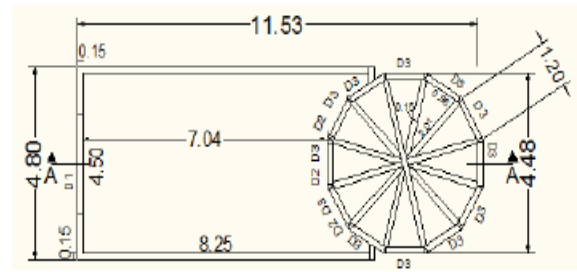

PLANTA COM DOZE DIVISÓRIAS

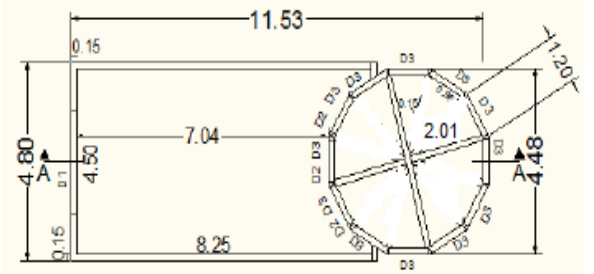

PLANTA COM QUATRO DIVISÓRIAS 


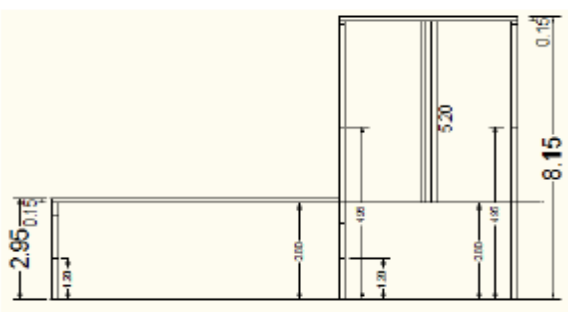

CORTE AA

\begin{tabular}{|c|c|c|c|}
\hline Abertura & O1 & O2 & O3 \\
\hline Largura (m) & 2.50 & 1.00 & 1.70 \\
\hline Altura (m) & 1.20 & 1.00 & 2.95 \\
\hline Peitoril $(\mathrm{m})$ & 1.20 & 1.20 & 4.95 \\
\hline Quantidade & 1 & 3 & 8 \\
\hline
\end{tabular}

Octodirecional

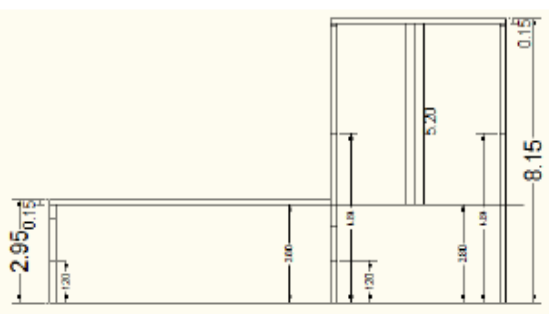

CORTE AA

\begin{tabular}{|c|c|c|c|}
\hline Abertura & D1 & D2 & D3 \\
\hline Largura (m) & 2.50 & 1.00 & 1.10 \\
\hline Altura (m) & 1.20 & 1.00 & 3.06 \\
\hline Peitoril (m) & 1.20 & 1.20 & 4.84 \\
\hline Quantidade & 1 & 3 & 12 \\
\hline
\end{tabular}

Dodecadirecional

Fonte: Andrade (2013)

Finalizando, o edifício e o domínio foram unidos em um único modelo, eliminando todos os elementos externos às fronteiras do domínio (Figura 4).

\section{Determinação da malha}

A malha é a discretização do espaço fluido dentro do domínio, e é gerada automaticamente pelo programa, por meio de um método iterativo, sendo refinada nas áreas com elevados gradientes (Figura 5).

\section{Figura 4 - Modelo do domínio e edificação com torre}

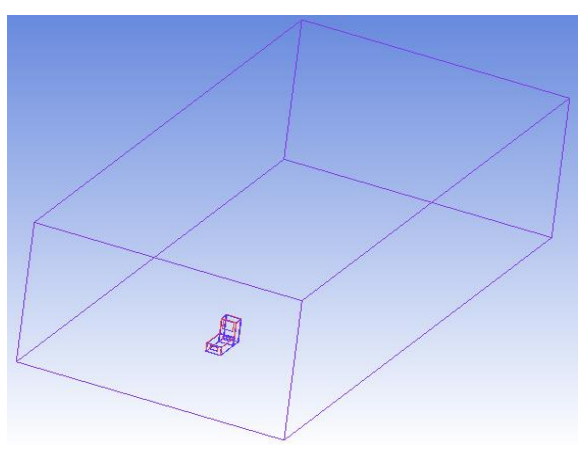

Figura 5 - Malha no domínio e na edificação com torre

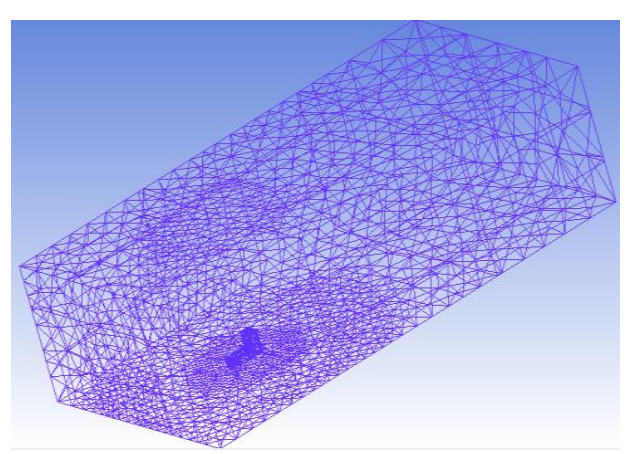

Fonte: Andrade (2013)

\subsection{Software CFX Pre ${ }^{\circledR}$}

As simulações foram realizadas em regime permanente, uma vez que as condições de contorno se mantêm constantes, alterando-se apenas a velocidade de entrada de ar no modelo para cada posição: baixa $(2 \mathrm{~m} / \mathrm{s})$, média $(5 \mathrm{~m} / \mathrm{s})$ e alta $(10 \mathrm{~m} / \mathrm{s})$, especificando-se o tipo de domínio como fluido (o ar é considerado gás ideal, a pressão atmosférica local, desprezando-se as variações de temperatura, e o modelo de turbulência, o modelo k- $\varepsilon$ (k-Epsilon)). 


\subsection{Software CFX Solver ${ }^{\circledR}$}

Após as etapas de elaboração do modelo iniciou-se a simulação propriamente dita no CFX-Solver ${ }^{\circledR}$.

\subsection{Software CFX Post ${ }^{\circledR}$}

Nesse trabalho são mostrados somente os gráficos obtidos por meio dos valores tabelados, gerados ao final de cada simulação, permitindo fazer uma análise do escoamento de forma rápida e direta.

\section{RESULTADOS E DISCUSSÃO}

\subsection{Simulações de Velocidade}

\section{Torre Octodirecional}

A Figura 6 representa a comparação entre os valores de velocidade do ar resultantes das simulações com as torres de oito (8d) e quatro (4d) divisórias.

Observa-se que os valores da velocidade do ar interno à edificação (Ved) obtidos nas simulações da torre com quatro divisórias são superiores aos da torre com oito divisórias, exceto na posição de $0^{\circ}$, na qual ocorre o contrário, fato provocado certamente pela abertura da edificação, que é favorável a entrada do ar em seu interior, e, com um maior número de divisórias, o ar demora mais tempo para se dispersar pelas aberturas da torre, aumentando assim a sua velocidade.

Verifica-se que os valores de velocidade do ar são maiores nas aberturas da torre posicionadas perpendicularmente ao vento, além disso, os valores das simulações com a torre de quatro divisórias superam aos da de oito divisórias na maioria das posições para as três velocidades ensaiadas, notadamente nas posições de $270^{\circ}$ para $\mathrm{V} 1 \mathrm{e} \mathrm{V8}, 0^{\circ}$ para $\mathrm{V} 2$ e V3, $90^{\circ}$ para V5 e $180^{\circ}$ para $\mathrm{V} 7$, mostrando que para essa torre a diminuição do número de divisórias promove maiores velocidades.

Figura 6 - Comparação dos valores de velocidade do ar entre os dois tipos de torre octodirecional

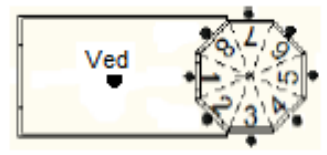

Pontos de velocidade medidos

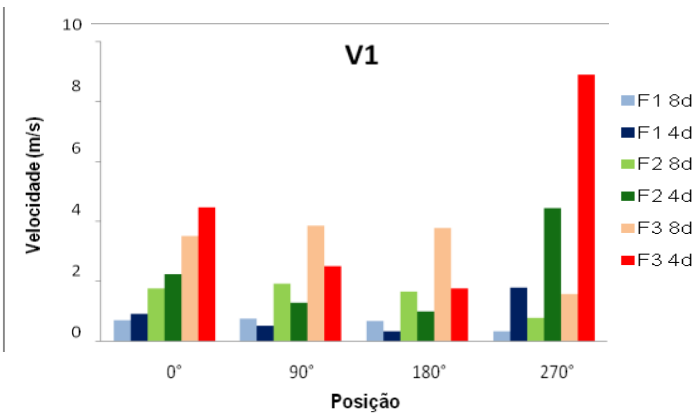

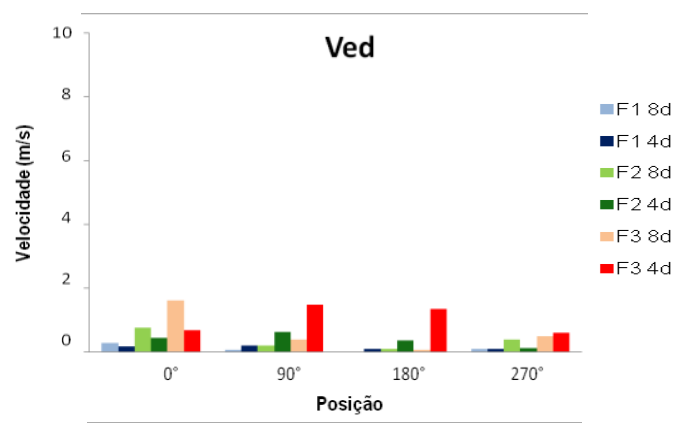

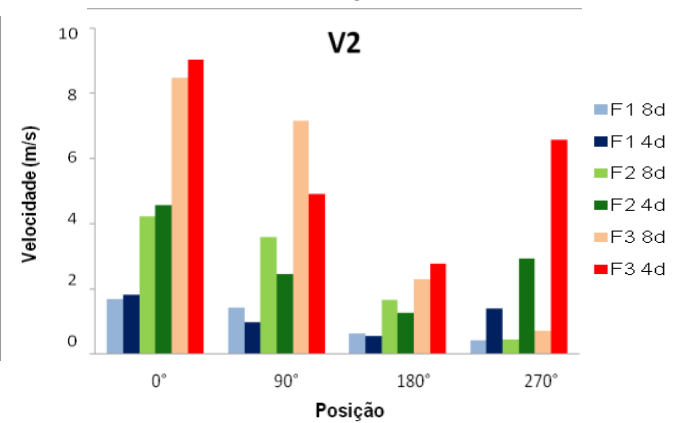



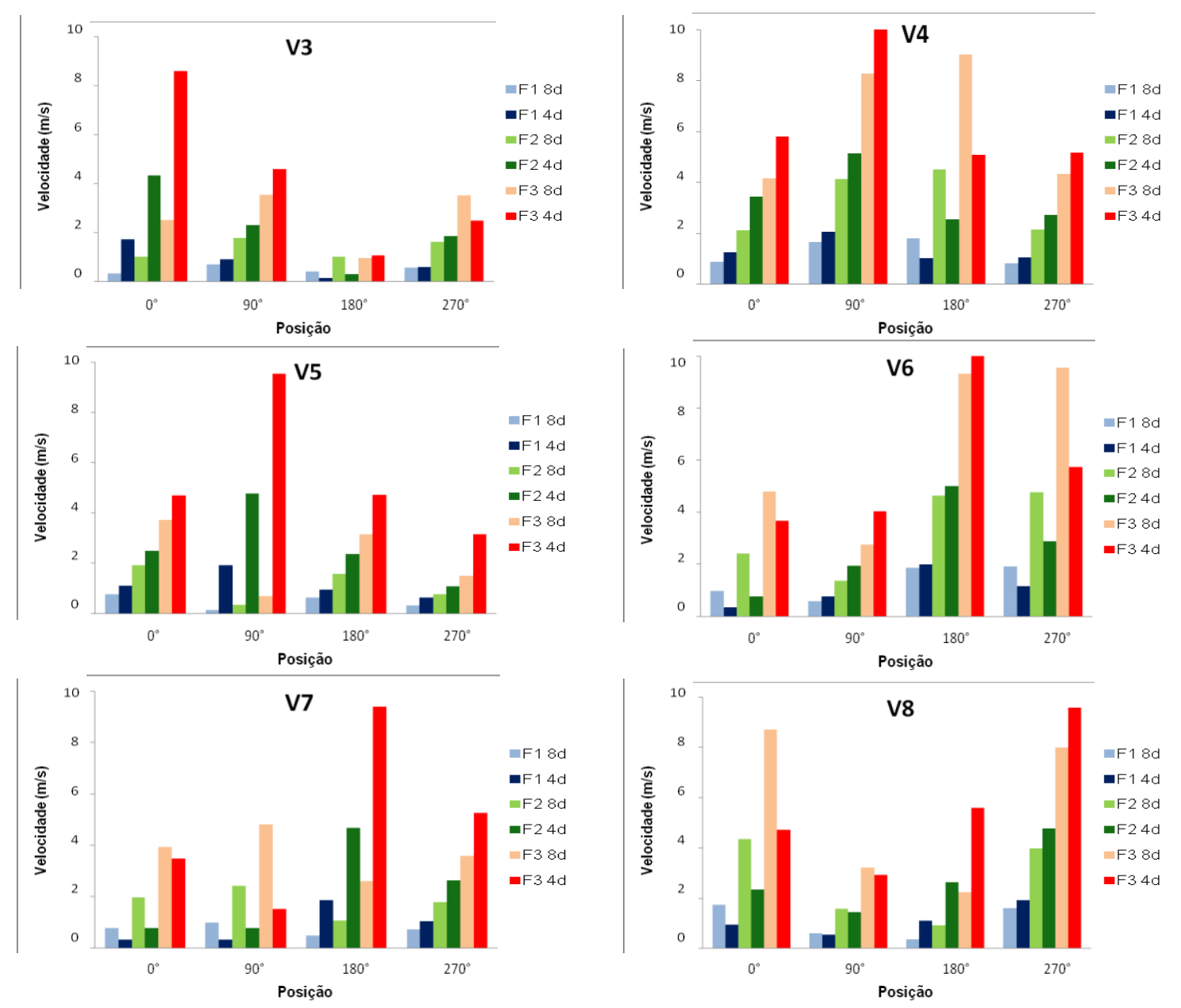

Ved: Velocidade do ar no interior da edificação / V1 a V8: Velocidades do ar nas aberturas das torres F1 8d, F2 8d, F3 8d: Velocidades simuladas na torre com 8 divisórias correspondendo respectivamente a $2 \mathrm{~m} / \mathrm{s}, 5 \mathrm{~m} / \mathrm{s}$ e $10 \mathrm{~m} / \mathrm{s}$

F1 4d, F2 4d, F3 4d: Velocidades simuladas na torre com 4 divisórias correspondendo respectivamente a $2 \mathrm{~m} / \mathrm{s}, 5 \mathrm{~m} / \mathrm{s}$ e $10 \mathrm{~m} / \mathrm{s}$

\section{Torre Dodecadirecional}

A Figura 7 representa a comparação entre os valores de velocidade do ar resultantes das simulações com as torres de doze (12d) e quatro (4d) divisórias.

Considerando os valores da velocidade do ar interno à edificação (Ved) para os dois casos, nota-se que tais valores são maiores para a torre com doze divisórias nas posições de $90^{\circ}$ e $270^{\circ}$, ao contrário das posições de $0^{\circ}$ e $180^{\circ}$, onde os maiores valores são da torre com quatro divisórias. Isso pode ser explicado por meio da entrada de ar pela abertura da edificação, sendo que nas posições de $0^{\circ}$ e $180^{\circ}$ o fluxo é maior, e, com um maior número de aberturas com dimensões menores que a octodirecional, o ar terá mais dificuldade para se dispersar, aumentando assim a sua velocidade.

Assim como na torre octodirecional, os valores de velocidade do ar nas aberturas dos dois tipos de torre dodecadirecional são maiores naquelas posicionadas perpendicularmente ao vento, e, observa-se, também, que esses valores na torre com quatro divisórias elevam-se quando comparados aos da torre com doze divisórias nas três velocidades simuladas, em mais da metade dos casos, principalmente nas posições de $0^{\circ}$ para $\mathrm{V} 1, \mathrm{~V} 4$ e $\mathrm{V} 6,90^{\circ}$ para $\mathrm{V} 7,180^{\circ}$ para $\mathrm{V} 10,270^{\circ}$ para $\mathrm{V} 2, \mathrm{~V} 10$ e $\mathrm{V} 11$, 
comprovando que a redução do número de divisórias acarreta maiores valores de velocidade do ar.

Figura 7 - Comparação dos valores de velocidade do ar entre os dois tipos de torre dodecadirecional

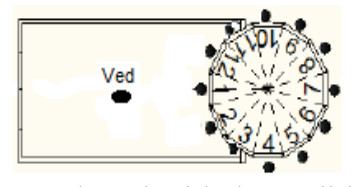

Pontos de velocidade medidos
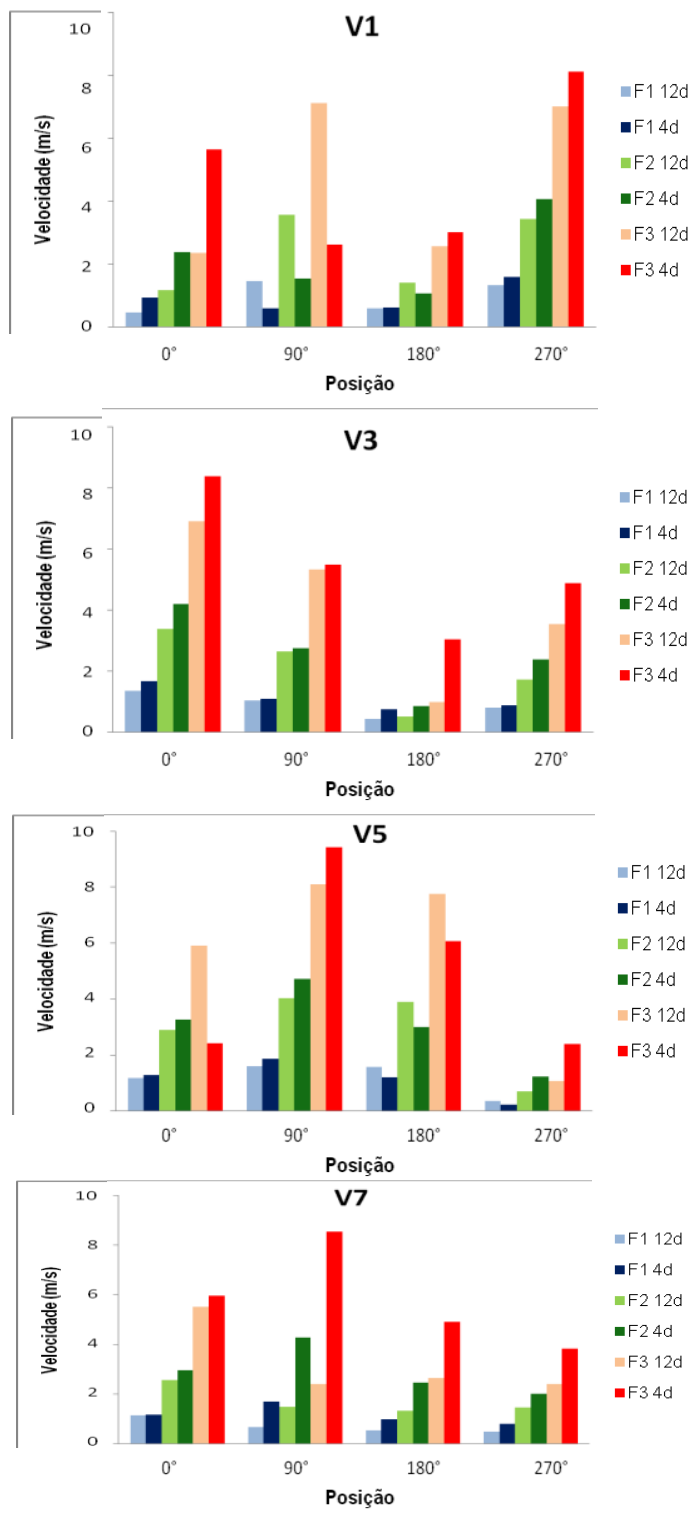
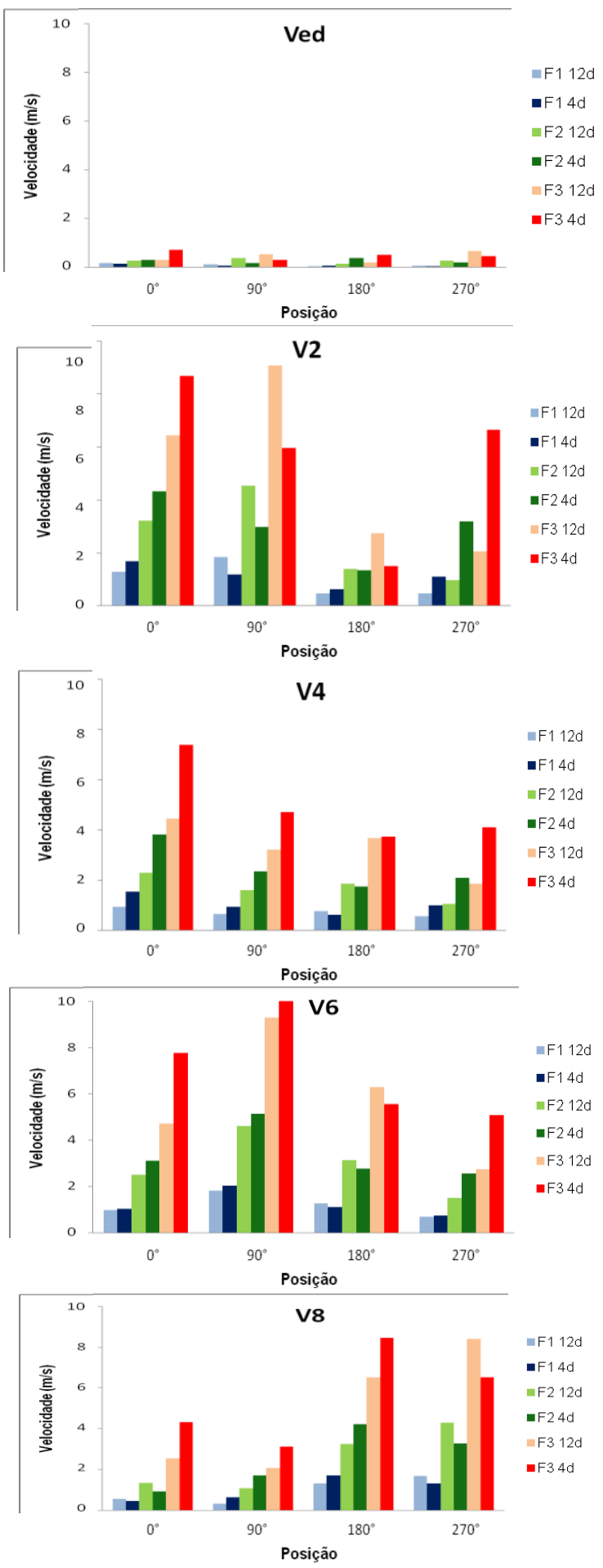

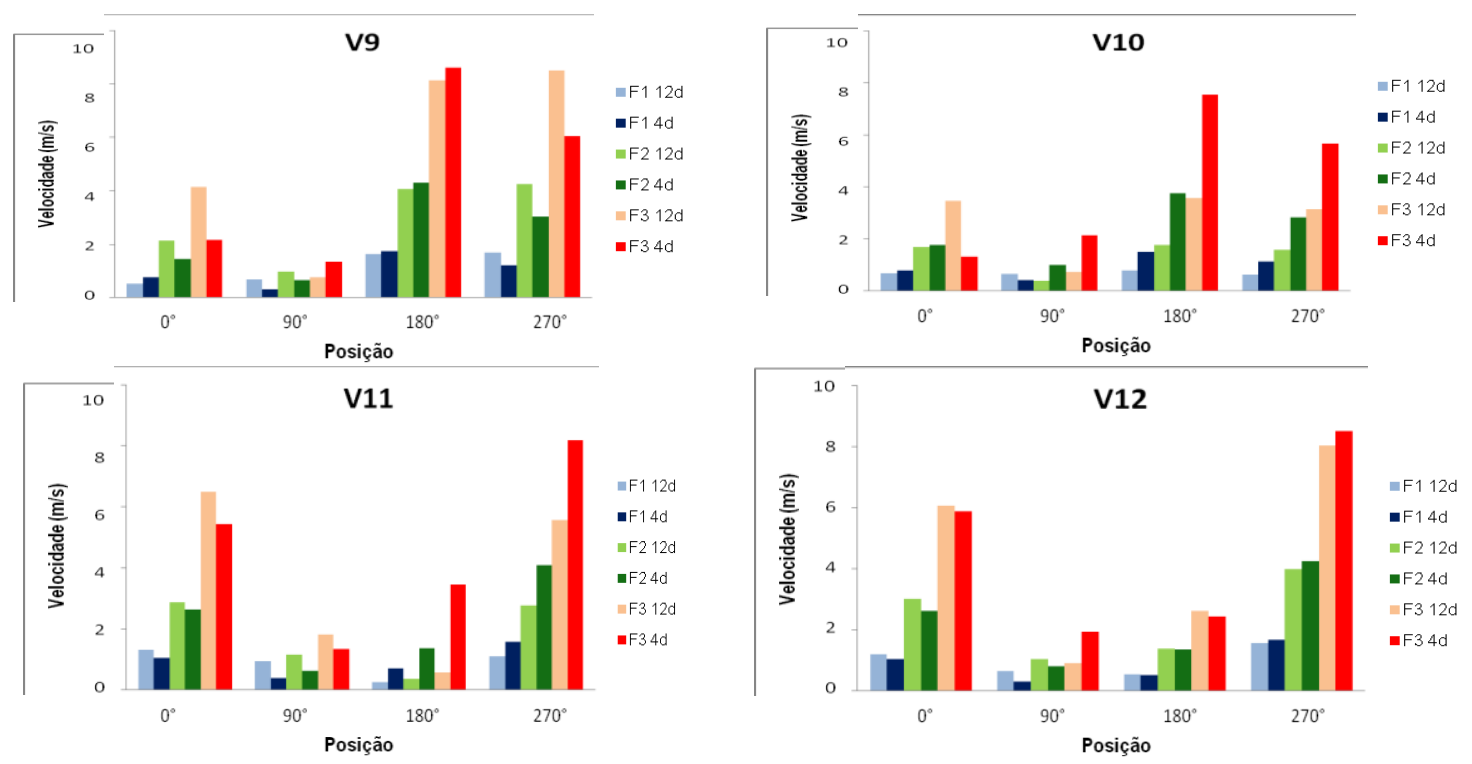

Ved: Velocidade do ar no interior da edificação / V1 a V12: Velocidades do ar nas aberturas das torres F1 12d, F2 12d, F3 12d: Velocidades simuladas na torre com 12 divisórias correspondendo respectivamente a $2 \mathrm{~m} / \mathrm{s}, 5 \mathrm{~m} / \mathrm{s}$ e $10 \mathrm{~m} / \mathrm{s}$

F1 4d, F2 4d, F3 4d: Velocidades simuladas na torre com 4 divisórias correspondendo respectivamente a $2 \mathrm{~m} / \mathrm{s}, 5 \mathrm{~m} / \mathrm{s}$ e $10 \mathrm{~m} / \mathrm{s}$

\subsection{Simulações de Pressão}

Os valores das pressões computadas nas simulações permitiram calcular o coeficiente de pressão (Cp), utilizando a fórmula (1)a inserida no CFX Post ${ }^{\circledR}$.

A tabela 1 mostra os sinais dos coeficientes de pressão $(\mathrm{Cp})$ para cada torre simulada, os quais indicam barlavento, quando a torre funciona como captador ( $\mathrm{Cp}$ positivo), ou sota-vento, quando a torre atua como extrator ( $\mathrm{Cp}$ negativo).

Analisando-se os valores mostrados nessa tabela observa-se que as torres possuem coeficientes de pressão negativos na maior parte das posições, destacando-se como extratores. Todavia, como a abordagem desse artigo é sobre captadores, então serão considerados apenas os Cps positivos.

Em ambas as torres, o número de Cps positivos é maior para os casos em que a quantidade de divisórias é menor, provando que essa redução aumenta a captação das torres.

A comparação entre o número de Cps positivos das duas torres com quatro divisórias permite afirmar que a dodecadirecional apresenta o maior número de Cps positivos, isto é, essa torre se destaca como captador em relação à outra.

Tabela 1 - Quantidade de coeficientes de pressão (Cp) nas torres analisadas

\begin{tabular}{|c|c|c|c|c|c|c|}
\hline $\begin{array}{c}\text { Sinais } \\
\text { X } \\
\text { Torres }\end{array}$ & $\begin{array}{l}\text { Número de } \\
\text { Divisórias }\end{array}$ & + & $\begin{array}{c}+ \\
(\%)\end{array}$ & - & $(\%)$ & Total \\
\hline \multirow[t]{2}{*}{ Octodirecional } & 4 & 39 & 41 & 57 & 59 & \multirow{2}{*}{96} \\
\hline & 8 & 33 & 34 & 63 & 66 & \\
\hline \multirow[t]{2}{*}{ Dodecadirecional } & 4 & 69 & 48 & 75 & 52 & \multirow{2}{*}{144} \\
\hline & 12 & 36 & 25 & 108 & 75 & \\
\hline
\end{tabular}




\section{CONCLUSÕES}

Comparando os quatro modelos de torres, quanto à velocidade do ar interno da edificação (Ved), conclui-se que as torres octodirecional e dodecadirecional com quatro divisórias são mais eficazes no aumento da ventilação interna, por apresentarem os maiores valores, principalmente a octodirecional, na maioria das posições para as três velocidades consideradas nas simulações.

$\mathrm{Na}$ análise de coeficientes de pressão $(\mathrm{Cp})$ positivos, notou-se o destaque das torres com quatro divisórias atuando como captadores. Embora a dodecadirecional apresente o maior número desses Cps (48\%), é a octodirecional que desempenha uma maior troca de ar por possuir maiores valores de velocidade do ar interno da edificação (Ved).

O uso de uma ferramenta CFD em simulações, como o software $\mathrm{CFX}^{\circledR}$, oferece grande auxílio no projeto de ventilação, e, os resultados fornecidos comprovam fenômenos conhecidos e esperados, além de identificar pontos de melhoria no projeto.

\section{REFERÊNCIAS}

ANDRADE, N.C. Estudo da ventilação natural em edificações com captadores de vento multidirecionais por meio de ensaios em túnel de vento e simulações computacionais. 2013. 301p. Tese (Doutorado em Engenharia Civil), Programa de Pós-Graduação em Engenharia Civil, Universidade Estadual de Campinas, Campinas, 2013.

BAHADORI, M.N. Viability of wind towers in achieving summer comfort in the hot arid regions of the Middle East. Renewable Energy, v. 5, n. 5-8, p. 879-892, Aug. 1994.

BAHADORI, M.N.; MAZIDI, M.; DEHGHANI, A.R. Experimental investigation of new designs of wind towers. Renewable Energy, v. 33, n. 10, p. 2273-2281, Oct. 2008.

FORTUNA, A. R. Técnicas computacionais para dinâmica dos fluidos: conceitos básicos e aplicações. São Paulo: EdUSP, 2000.

LIDDAMENT, M. W. Air Infiltration Calculation Techniques: an applications guide. Bracknell: AIVC, 1986.

MARTINS, T.A.L.; BASTOS, L.E.G.; BARROSO-KRAUSE, C.; BITTENCOURT, L.S.; PASSOS, I. O uso de torres de vento em edificações residenciais geminadas de meia morada. In: ENCONTRO NACIONAL DE CONFORTO NO AMBIENTE CONSTRUÍDO, 10., 2009, Natal. Anais... Porto Alegre: ANTAC, 2009.

OLIVEIRA, B.Y.; MARIANO, G.C.; QUADRI, M.B. Um estudo CFD de vazamento de óleo a partir de dutos submersos. In: CONGRESSO BRASILEIRO DE ENGENHARIA QUÍMICA EM INICIAÇÃO CIENTÍFICA, 8., 2009, Uberlândia. Anais... São Paulo: ABEQ, 2009. Disponível em: <www.cobeqic2009.feq.ufu.br/uploads/media/99517091.pdf>. Acesso em: 23 mar. 2010.

VAN LENGEN, J. Manual do arquiteto descalço. Tradução de Valdo de Freitas Felinto. Porto Alegre: Livraria do Arquiteto, 2004. 